\title{
Aktuelle Aspekte bei der Diagnostik und Therapie des Plasmazellmyeloms
}

\section{Current Aspects in Diagnosis and Therapy of Plasma Cell Myeloma}

Autoren

Marc-Andrea Bärtsch ${ }^{1}$, Hartmut Goldschmidt ${ }^{1,2}$

Institut

1 Medizinische Klinik V, Universitätsklinikum Heidelberg

2 Nationales Centrum für Tumorerkrankungen NCT Heidelberg

Bibliografie

DOI http://dx.doi.org/10.1055/s-0043-100295

Dtsch Med Wochenschr 2017; 142: 800-804

(C) Georg Thieme Verlag KG Stuttgart · New York

ISSN 0012-0472

\section{WAS IST NEU?}

Definition, Inzidenz und klinisches Bild Die mediane Überlebenszeit mit Multiplem Myelom (MM) hat sich in den letzten 15 Jahren verdoppelt.

Diagnostik Die Diagnosekriterien des therapiepflichtigen MM wurden 2014 um Biomarker erweitert.

Erstlinientherapie Fitte, junge Patienten sind mit einer Dreierkombination zu behandeln. Die Hochdosistherapie ist weiterhin ein wesentliches Element der Initialtherapie junger MM-Patienten.

Rezidivtherapie In den letzten 2 Jahren wurden 5 neue Substanzen zur Behandlung des rezidivierten MM zugelassen.

Ausblick Remissionstiefe und Immunonkologie gewinnen zunehmend an Bedeutung in der Therapie des MM.

\section{Definition, Inzidenz, klinisches Bild}

Das Plasmazellmyelom (WHO-Definition) wird in der klinischen Routine meist als Multiples Myelom (MM) bezeichnet. Das MM ist eine maligne Erkrankung der Plasmazellen, welche die am weitesten differenzierten Zellen der B-Lymphozyten-Reihe sind. Durch die monoklonale Plasmazellvermehrung im Knochenmark werden komplette und/oder inkomplette Immunglobuline (Leichtketten) vermehrt produziert. Die monoklonalen Immunglobuline lassen sich im Serum und/oder Urin als "M-Gradient“ ( $\triangleright$ Abb. 1) bzw. im Serum mittels des freien Leichtkettentests quantifizieren. Das symptomatische MM ist eine Systemerkrankung und durch multiple Osteolysen, Nierenfunktionsverschlechterung, Anämie und/oder Hyperkalzämie gekennzeichnet. Seit 2014 werden auch Biomarker für die Diagnosesicherung des symptomatischen MM genutzt ( $\vee$ Tab. 1). Abzugrenzen ist das solitäre Plasmozytom, welches durch einen isolierten ossären oder extraossären Plasmazelltumor definiert ist.

Das MM ist die zweithäufigste hämatologische maligne Erkrankung in den westlichen Industriestaaten. Die Inzidenz liegt bei ca. 6 Neuerkrankungen pro 100000 Einwohner in Europa [1]. Patienten sind zum Zeitpunkt der Diagnose im Median ca. 70 Jahre alt. Die mittlere Überlebenszeit konnte in den letzten 15 Jahren verdoppelt werden. So überleben Patienten nach Hochdosistherapie im
Median 8-10 Jahre; Patienten, die nicht intensiv behandelt werden können ca. 5 Jahre.

Die Symptome des MM sind unspezifisch. $80 \%$ der Patienten klagen über Knochenschmerzen. Weitere häufige Symptome sind Anämie (50\%), Nierenfunktionsverschlechterung (20\%) und Hyperkalzämie (bis zu $15 \%$ ). Die Zeit bis zur Diagnosesicherung nach dem Auftreten der ersten Symptome beträgt im Median 3 Monate. Alle Patienten mit MM haben in Ihrer Vorgeschichte eine Monoklonale Gammopathie unbestimmter Signifikanz (MGUS) und/oder ein Smoldering Multiples Myelom (SMM), welche aber aufgrund fehlender Symptomatik oftmals nicht diagnostiziert wurden. Die Differenzierung zwischen MGUS, SMM und MM ist in - Tab. 1 dargestellt.

\section{Klinische Relevanz}

Bei unspezifischen Symptomen wie Knochenschmerzen, Anämie, Nierenfunktionsverschlechterung und/ oder Hyperkalziämie sollte ein MM ausgeschlossen werden.

\section{Diagnostik}

Für die Diagnosesicherung des MM sind serologische Untersuchungen, eine Knochenmarkpunktion bzw. eine Tumorbiopsie und eine Skelettbildgebung erforderlich (Box 1). Die Kriterien der International Myeloma Working 


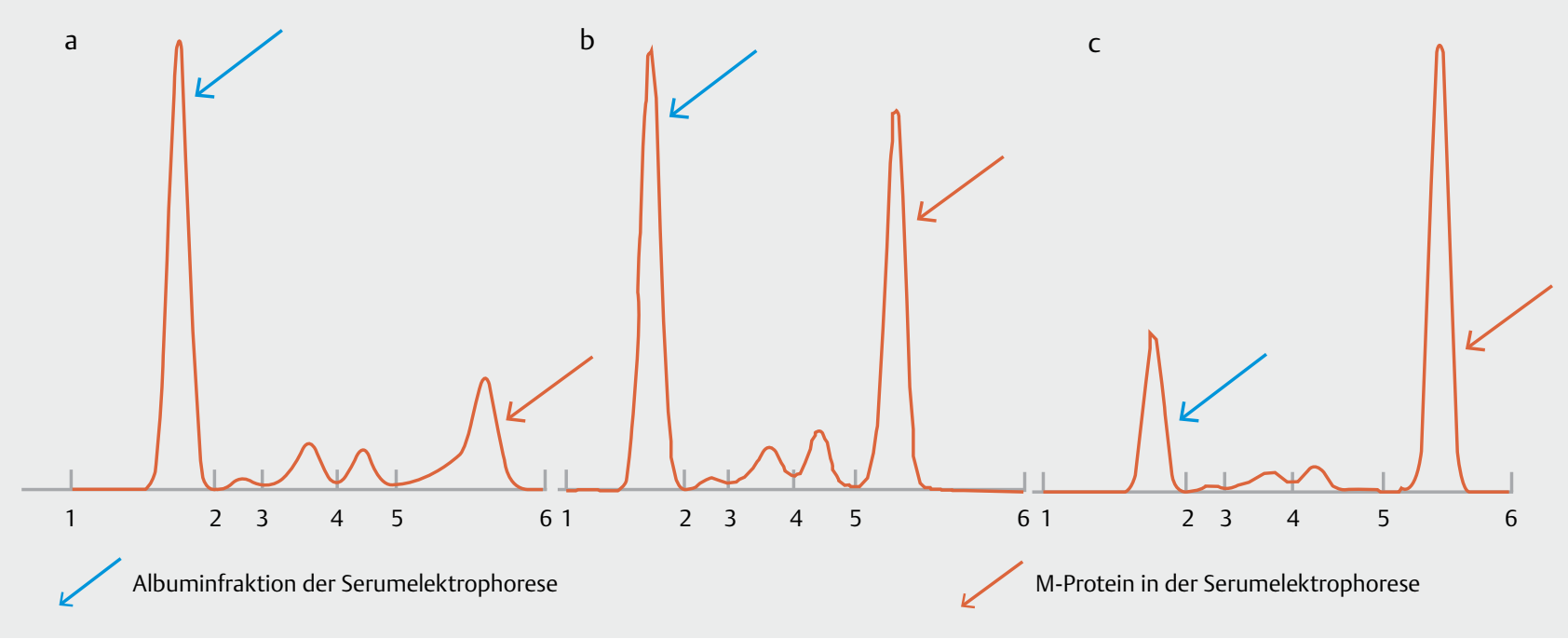

- Abb. 1 Beispiele für M-Gradienten in der Elektrophorese bei a monoklonaler Gammopathie unklarer Signifikanz, b Smoldering Multiplem Myelom und c multiplem Myelom.

- Tab. 1 Diagnosekriterien für die Monoklonale Gammopathie unklarer Signifikanz (MGUS), das Smoldering Multiple Myelom und das Multiple Myelom der International Myeloma Working Group [2, 3].

\begin{tabular}{|c|c|c|c|}
\hline & MGUS & Smoldering MM & MM (behandlungspflichtig) \\
\hline \multirow[t]{4}{*}{ monoklonales Protein } & $<30 \mathrm{~g} / \mathrm{l}$ im Serum & $\geq 30 \mathrm{~g} / \mathrm{l} \mathrm{im} \mathrm{Serum}$ & vorhanden im Serum und/oder Urin \\
\hline & und & und/oder & \\
\hline & $<500 \mathrm{mg} /$ Tag im Urin & $>500 \mathrm{mg} /$ Tag im Urin & \\
\hline & und & und/oder & und/oder \\
\hline \multirow{2}{*}{$\begin{array}{l}\text { prozentualer Anteil der monoklona- } \\
\text { len Plasmazellen im Knochenmark }\end{array}$} & $<10 \%$ & $\geq 10 \%-60 \%$ & $\geq 10 \%$ oder Plasmozytom \\
\hline & und & und & und \\
\hline Organschädigung & keine & keine & SLIM-CRAB-Kriterien erfüllt \\
\hline
\end{tabular}

\section{SLIM-CRAB-Kriterien}

Endorganschäden (mindestens eines der folgenden vier): $C=$ Hyperkalzämie (Konzentration im Serum $>11 \mathrm{mg} / \mathrm{dl}$ oder $0,25 \mathrm{mmol} / \mathrm{l}$ über dem Normwert); $\mathrm{R}=$ Niereninsuffizienz (Kreatinin $>2 \mathrm{mg} / \mathrm{dl}$ oder Kreatinin Clearance $<40 \mathrm{ml} / \mathrm{min}$ ); A = Anämie (Hämoglobin $<10 \mathrm{~g} / \mathrm{dl}$ oder $>2 \mathrm{~g} / \mathrm{dl}$ unter dem Normwert); B = Knochenerkrankung (eine oder mehrere Osteolysen nachgewiesen durch Projektionsradiografie, CT oder PET-CT). und/oder

Biomarker (mindestens ein Biomarker nachweisbar): Anteil der klonalen Plasmazellen im Knochenmark $\geq 60 \%$, Verhältnis von beteiligten zu unbeteiligten freien Leichtketten im Serum $\geq 100$ (Werte basieren auf dem serum Freelite assay von Binding Site) und betroffene freie Leichtketten mit einer Konzentration von $\geq 100 \mathrm{mg} / \mathrm{l}$, mehr als eine fokale Läsion im MRT $\geq 5 \mathrm{~mm}$.

Group zur Diagnose des MM wurden 2014 aktualisiert [2, 3]. Bei dieser Aktualisierung wurden die klassischen CRAB-Kriterien um sogenannte „Biomarker“ zu den heute gültigen SLIM-CRAB-Kriterien erweitert ( $>$ Tab. 1). Diese Biomarker kündigen mit einer Wahrscheinlichkeit von $>80 \%$ das Auftreten von Symptomen, bedingt durch Organschäden, innerhalb der folgenden 2 Jahre an. Die Diskussion hinsichtlich Überbehandlung versus Kuration des MM im frühen Stadium wird aktuell kontrovers geführt. Deshalb sollten Patienten mit SLIM-CRAB-Kriterien möglichst in prospektiven Registern oder Therapiestudien erfasst bzw. behandelt werden. Das SMM ist nach den aktuellen Kriterien nicht zu behandeln. Weltweit sind Studien aktiv, welche den sehr frühen Therapiebeginn beim SMM prospektiv untersuchen. 


\section{BOX 1}

Labor- und Knochenmarkdiagnostik sowie Bildgebung beim Multiplen Myelom zum Zeitpunkt der Diagnosestellung

Labordiagnostik

- peripheres Blut: Blutbild mit Differenzialblutbild

- biochemische Parameter: Elektrolyte (Natrium, Kalium, Kalzium), glomeruläre Filtrationsrate, Kreatinin, Harnstoff, Harnsäure, C-reaktives Protein (CRP), Laktatdehydrogenase (LDH)

- Beta-2-Mikroglobulin, Gesamteiweiß, Albumin, Immunglobuline quantitativ (IgA, IgG, IgM), Glutamat-Oxalacetat-Transaminase (GOT), Glutamat-Pyruvat-Transaminase (GPT), Bilirubin, alkalische Phosphatase (AP)

- Serumproteinelektrophorese, Immunfixationselektrophorese

- Bestimmung freier Leichtketten im Serum (Freelite ${ }^{\circledR}$ Test)

- Urinuntersuchungen (24 Stunden Sammelurin): Kreatinin-Clearence, Urinproteinelektrophorese oder quantitative Leichtkettenbestimmung (kappa und lambda), Immunfixationselektrophorese, Gesamtprotein, Albumin

Knochenmarkdiagnostik

- Aspirationszytologie

- Histologie (mit immunhistochemischer Sicherung der Leichtkettenrestriktion)

- molokulare Zytogenetik an CD138-angereicherten Plasmazellen mithilfe der Fluoreszenz In-situHybridisierung (FISH) z. B.: $t(4 ; 14), t(14 ; 16)$, del (17p13), + 1q21 > 3 Kopien $\rightarrow$ ungünstige Prognose

\section{Bildgebung}

- Low-dose-Ganzkörper-Computertomographie (ohne Kontrastmittel) zur Diagnostik von Osteolysen, Osteopenie und zur Stabilitätsbeurteilung

- MRT bei Verdacht auf extramedulläre Manifestationen und zur Abklärung der Behandlungsbedürftigkeit eines SMM

Hinsichtlich der Bildgebung ist zu betonen, dass in Deutschland die Niedrig-Dosis-CT weitgehend flächendeckend realisierbar ist. Die MRT kann bei frühen Stadien und negativem Niedrig-Dosis-CT-Befund weiterführende Informationen erbringen. Insbesondere fokale Läsionen im Knochenmark korrelieren mit einer schnellen Symptomentwicklung. Deshalb sind eindeutige multiple fokale Läsionen in der MRT eine Behandlungsindikation.

Für die Knochenmarkdiagnostik ist eine Klassifikation der Zellen in unreife oder reife Plasmazellen zu empfehlen.
Die aus dem Knochenmark aspirierten Myelomzellen sind insbesondere bei Eignung für eine intensive Therapie hinsichtlich zytogenetischer Aberrationen zu untersuchen. Prognostisch ungünstige zytogenetische Aberrationen sind 4;14- oder 14;16-Translokationen, die 17pDeletion oder das Vorliegen von mehr als 3 Kopien der 1q21-Amplifikation. Erstmalig werden Therapien an zytogenetische Risikomuster gekoppelt. Zum Beispiel profitieren Hochrisiko-Patienten von einer langandauernden Proteasominhibitor-basierten Therapie [4].

Um die Prognose zu beschreiben, sind verschiedene Stadieneinteilungen des MM publiziert. Die am längsten etablierte Einteilung ist die Stadieneinteilung nach dem International Staging System (ISS) basierend auf den Serumwerten für Albumin und Beta-2-Mikroglobulin. Zu diesen serologischen Parametern kann die Zytogenetik addiert werden, sodass eine bessere Diskriminierung der Risikogruppen erfolgt. Eine weitere Möglichkeit ist die zusätzliche Implementierung des LDH-Werts, sodass mit dem revidierten R-ISS eine zuverlässige Prognoseabschätzung möglich ist [5].

\section{Erstlinientherapie}

Symptomatische Myelompatienten sind umgehend zu behandeln. Patienten mit positiven Biomarkern als alleinigem Therapiekriterium sind über die Prognose und mögliche Komplikationen aufzuklären. In der klinischen Praxis kann bei Unsicherheit über die Therapieindikation abgewartet werden. So sind bei unklaren Läsionen in der Kernspintomografie Folgeuntersuchungen nach 3-6 Monaten sinnvoll. Treten neue Läsionen auf oder vergrößern sich bekannte Läsionen, so ist die Therapieindikation als gesichert anzusehen. Bei Patienten mit einem Leichtkettenquotienten $\geq 100$ und einer absoluten Konzentration der beteiligten Leichtkette von $\geq 100 \mathrm{mg} / \mathrm{dl}$ sollte eine erneute Messung im Verlauf zum Ausschluss von Messungenauigkeiten erfolgen. Patienten mit hoher Compliance (d. h. Patienten, die mehr als 2 Liter am Tag trinken, keine nicht-steroidalen Antirheumatika einnehmen und bei Fieber frühzeitig den Arzt aufsuchen) können beobachtet werden. So sind Patienten bekannt, die aufgrund eines positiven SLIM-Kriteriums als behandlungspflichtig anzusehen sind, bei denen über viele Jahre kein Endorganschaden aufgetreten ist.

Bei einer positiven Therapieentscheidung ist umgehend mit dem Patienten zu besprechen, welche Therapieoption gewählt wird. Zu unterscheiden ist zwischen der intensiven Behandlung, welche eine Hochdosistherapie gefolgt von einer autologen Blutstammzelltransplantation beinhaltet und einer Behandlung ohne Hochdosistherapie. Ein transplantationserfahrener Kollege sollte möglichst in die Entscheidung einbezogen werden, da durch den Patienten die Risiken der Hochdosistherapie 
oft als sehr belastend angesehen werden. Einen Überblick über die primäre Therapieentscheidung gibt $>$ Abb. 2 .

Für fitte Patienten bis zum Alter von 70 Jahren, wird heute die Hochdosistherapie mit $200 \mathrm{mg} / \mathrm{m}^{2}$ Melphalan empfohlen. Die Tumormassenreduktion vor Hochdosistherapie erfolgt durch eine Induktionstherapie. Hier werden Dreierkombinationen bevorzugt. In Deutschland ist eine Kombination mit Bortezomib/Cyclophosphamid/Dexamethason oder Bortezomib/Lenalidomid/Dexamethason (im Rahmen der integrierten Versorgung umsetzbar) als beste Option anzusehen. Nach der Stammzellsammlung wird einmalig transplantiert. Gegenwärtig ist die Diskussion über die Tandemtransplantation nicht abgeschlossen. Wir empfehlen bei Nichterreichen mindestens einer nahezu kompletten Remission außerhalb von Studien eine 2. Hochdosistherapie, um die Remissionstiefe zu erhöhen.

Die Therapieoptionen nach Hochdosistherapie werden unterschiedlich gewertet. Außerhalb von Studien sollte für Patienten mit Resterkrankung und günstiger Zytogenetik eine Erhaltungstherapie erfolgen. Die Behandlung mit Thalidomid 50 mg wird in diesem Fall von den Krankenkassen in Deutschland erstattet. Patienten mit zytogenetischer Hochrisikokonstellation sollten über zwei Jahre mit Bortezomib 1,3 mg/m² alle zwei Wochen behandelt werden, hier muss die Kostenübernahme jedoch bei der Krankenkasse beantragt werden.

Für Patienten, die älter als 70 Jahre sind, wird in der Regel mit modernen Medikamenten primär behandelt. Für Patienten in gutem Allgemeinzustand ist eine Dreierkombination, z. B. mit Bortezomib/Melphalan/Prednisolon oder Bortezomib/Cyclophospamid/Dexamethason eine gute Option. Für Patienten in eingeschränktem Allgemeinzustand ist die weniger belastende Langzeittherapie mit Lenalidomid/Dexamethason als Primärtherapie zu empfehlen.

\section{Klinische Relevanz}

Teile der Erstlinientherapie beim MM sollten an Risikound Remissionsstatus des Patienten angepasst werden.

\section{Rezidivtherapie}

Meist wird ein Krankheitsprogress oder ein Rezidiv laborchemisch diagnostiziert. Bei einer Verdopplung des monoklonalen Proteins in kurzer Zeit (ca. 3 Monate) sollte mit einer systemischen Therapie begonnen werden. Werden CRAB-Kriterien nachgewiesen, so ist die Rezidivtherapieindikation eindeutig gegeben. Die Entscheidung, wann mit der Rezidivtherapie bei serologisch progredienten Patienten begonnen werden sollte, ist individuell zu treffen. Organschädigungen (z. B. Frakturen) sollten vermieden werden.

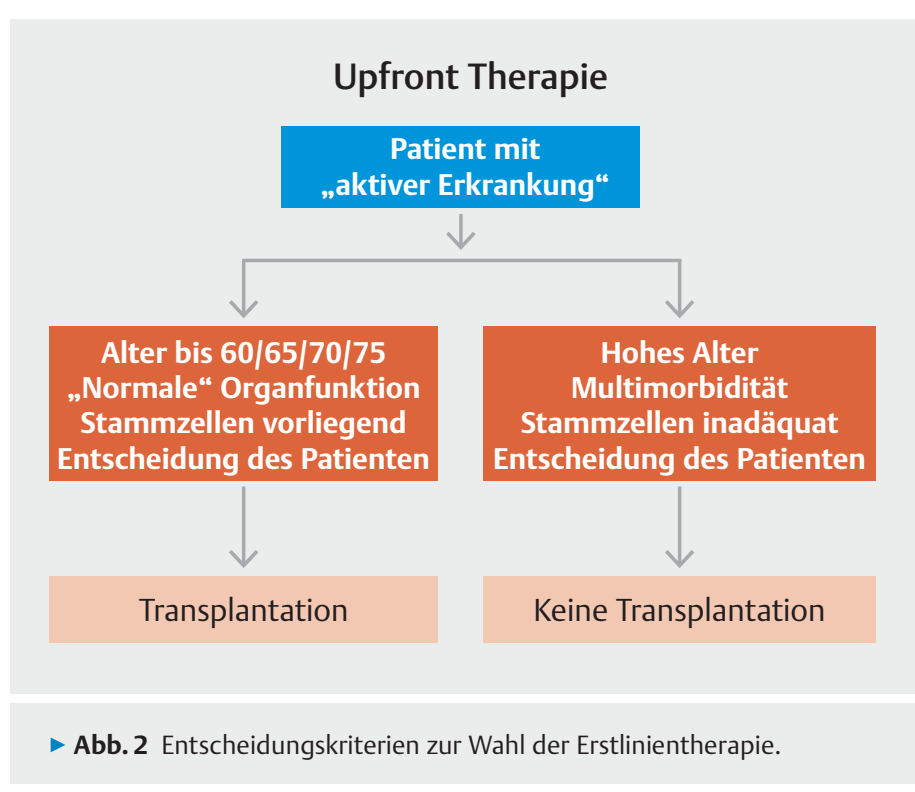

Die Wahl der Rezidivtherapie erfolgt hauptsächlich anhand von Patientenfaktoren, wie Alter, Komorbiditäten und Patientenpräferenzen sowie unter Berücksichtigung der Vortherapien (Effektivität, Nebenwirkungen, Dauer des Therapieansprechens). In den letzten 2 Jahren wurden erfreulicherweise 5 neue Substanzen zur Rezidivtherapie des MM zugelassen ( $>$ Tab. 2) [6]. Eine gesicherte Sequenz der Rezidivtherapien kann im Augenblick nicht empfohlen werden, da kein „Head-to-head-Vergleich“ der neuen Substanzen umfassend erfolgt ist. Eine Ausnahme stellt Carfilzomib dar, welches eine therapeutische Überlegenheit gegenüber Bortezomib in der Endeavor-Studie zur Rezidivtherapie zeigte.

\section{Klinische Relevanz}

Die Auswahl der Rezidivtherapie sollte in Abwesenheit evidenzbasierter Sequenzen unter Berücksichtigung von Patientenfaktoren und Vortherapien erfolgen.

\section{Ausblick}

Die klonale Genese des MM wird intensiv untersucht. Aufbauend auf dem erweiterten Verständnis der Pathogenese werden neue Therapieformen entwickelt. Auch beim MM wird die Remissionstiefe durch neue Medikamente oder Medikamentenkombinationen gesteigert. Begleitend sind Methoden der Messung der sogenannten minimalen Resterkrankung (MRD, minimal residual disease) weiterzuentwickeln. Die klassische Chemotherapie zur Behandlung des MM wird weitgehend verlassen. Neue Therapieoptionen wie Proteasominhibiton, DeacetylaseHemmung und Immunmodulation haben zunehmend an Bedeutung gewonnen. Zudem hat die Verfügbarkeit von Antikörpern zur Therapie des MM das therapeutische Arsenal entscheidend erweitert. Weitere Formen der Immuntherapie sind in frühen Studien mit erfolgverspre- 
- Tab. 2 Beispiele für neu zugelassene Arzneimittel oder Erweiterung der Zulassung (Indikation MM) seit 2015.

\begin{tabular}{|l|l|}
\hline Wirkstoff & Indikation \\
\hline Carfilzomib & $\begin{array}{l}\text { Kombination mit Dexamethason/Lenalidomid oder Dexamethason mono zur Behandlung von erwachsenen Patienten mit MM, } \\
\text { die mindestens eine Vortherapie erhalten haben. }\end{array}$ \\
\hline Lenalidomid & $\begin{array}{l}\text { Patienten mit unbehandeltem MM, die nicht transplantierbar sind. Kombination mit Dexamethason bei Patienten, die } \\
\text { mindestens eine vorausgegangene Therapie erhalten haben. }\end{array}$ \\
\hline Panobinostat & $\begin{array}{l}\text { Kombination mit Dexamethason und Bortezomib bei Patienten, mit mindestens zwei Vortherapien, darunter Bortezomib und } \\
\text { eine immunmodulierende Substanz. }\end{array}$ \\
\hline Daratumumab & \begin{tabular}{l} 
Monotherapie bei Erwachsenen mit rezidiviertem und refraktärem MM, die bereits mit einem Proteasominhibitor und einem \\
\hline Immonmodulator behandelt wurden und welche während der letzten Therapie Krankheitsprogression zeigten.
\end{tabular} \\
\hline Elotuzumab & $\begin{array}{l}\text { Kombination mit Dexamethason und Lenalidomid bei erwachsenen Patienten, die mindestens eine Vortherapie erhalten haben. } \\
\text { vorherige Therapie erhalten haben. }\end{array}$ \\
\hline Ixazomib & \\
\hline
\end{tabular}

chenden Resultaten gestartet. Es ist zu erwarten, dass die „Immun-Onkologie“ auch beim MM einen wichtigen Schritt in Richtung Heilung beitragen wird.

\section{Klinische Relevanz}

Die bereits zugelassenen neuen Therapeutika haben zu einer eindrücklichen Prognoseverbesserung für Patienten mit MM geführt; die konsequente Weiterentwicklung neuer Therapieansätze stimmt für die Zukunft weiter optimistisch.

\section{Interessenkonflikt}

Die Autoren geben an, dass kein Interessenkonflikt besteht.

\section{Über die Autoren}

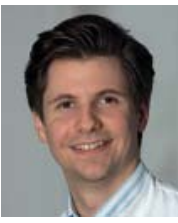

\section{Dr. Marc-Andrea Bärtsch}

ist Assistenzarzt an der Medizinischen Klinik V für Hämatologie, Onkologie und Rheumatologie, Universitätsklinikum Heidelberg. marc-andrea.baertsch@med.uni-heidelberg.de

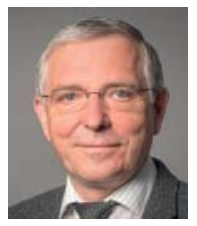

\section{Prof. Dr. Hartmut Goldschmidt}

ist Leiter der Sektion Multiples Myelom der Medizinischen Klinik $\vee$ und des Nationalen Centrums für Tumorerkrankungen. hartmut.goldschmidt@med.uni-heidelberg.de

\section{Korrespondenzadresse}

\section{Prof. Dr. Hartmut Goldschmidt}

Medizinische Klinik V, Universitätsklinikum Heidelberg

Im Neuenheimer Feld 410

69120 Heidelberg

hartmut.goldschmidt@med.uni-heidelberg.de

\section{Literatur}

[1] Katalinic A, Pritzkuleit R. Hochrechnung des institut für Krebsepidemiologie e.V., Lübeck für icd10: C90 auf Basis der Daten der Krebsregister BY, BR, HB, HH, MV, NI, NW (Reg.Bez. Münster) SL, SN, SH (2005-2009). Im Internet: http://www. GEKID.de; Stand: Januar 2016

[2] International Myeloma Working Group. Criteria for the classification of monoclonal gammopathies, multiple myeloma and related disorders: a report of the International Myeloma Working Group. Br J Haematol 2003; 121: 749-757

[3] Rajkumar SV, Dimopoulos MA, Palumbo A et al. International Myeloma Working Group updated criteria for the diagnosis of multiple myeloma. Lancet Oncol 2014; 15: e538-e548

[4] Neben K, Lokhorst HM, Jauch A et al. Administration of bortezomib before and after autologous stem-cell transplantation improves outcome in multiple myeloma patients with deletion 17p. Blood 2012; 119: 940 - 948

[5] Palumbo A, Avet-Loiseau H, Oliva S et al. Revised International Staging System for Multiple Myeloma: A Report From International Myeloma Working Group. J Clin Oncol 2015; 33: 2863 2869

[6] Bärtsch MA. Neue Therapieoptionen für Patienten mit rezidiviertem Multiplem Myelom. Karger Kompass Onkol 2016; 3 : 92-95 